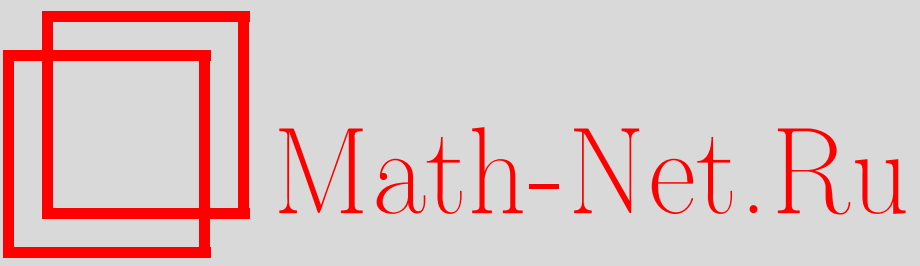

Г. Г. Козлов, Статистика краевой функции Грина одномерной разупорядоченной системы с бинарным или равномерным диагональным беспорядком, ТМ $\Phi, 2004$, том 140, номер 2, 337-352

DOI: https://doi.org/10.4213/tmf90

Использование Общероссийского математического портала Math-Net.Ru подразумевает, что вы прочитали и согласны с пользовательским соглашением

http://www . mathnet.ru/rus/agreement

Параметры загрузки:

IP: 3.80 .253 .173

26 апреля 2023 г., 12:29:17 


\section{СТАТИСТИКА КРАЕВОЙ ФУНКЦИИ ГРИНА ОДНОМЕРНОЙ РАЗУПОРЯДОЧЕННОЙ СИСТЕМЫ С БИНАРНЫМ ИЛИ РАВНОМЕРНЫМ ДИАГОНАЛЬНЫМ БЕСПОРЯДКОМ}

Для одномерной диагонально разупорядоченной цепочки исследована статистика краевой функции Грина (КФГ), построенной по случайной матрице гамильтониана этой системы. Беспорядок предполагается бинарным или равномерным. Показано, что в случае бинарного беспорядка функция распределения КФГ не является аналитической. Предложен простой алгоритм построения этой функции. Для случая равномерного беспорядка функция распределения КФГ вычислена точно на некотором интервале, а для полного ее определения и вычисления усредненной К $\Phi$ предложен простой и эффективный метод. Все полученные результаты проверены с помощью прямой компьютерной диагонализации, обнаружено хорошее согласие.

Ключевые слова: разупорядоченная система, случайная матрица, функция Грина, плотность состояний.

\section{1. ВВЕДЕНИЕ, ПОСТАНОВКА ЗАДАЧИ И ОСНОВНЫЕ РЕЗУЛЬТАТЫ}

К разупорядоченным обычно относят системы, не имеюшие транслящионной симметрии. Так как в реальных физических системах всегда следует ожидать нарушения этой симметрии, то можно сказать, что упорядоченные системы и соответствующие им математические модели являются первым и не всегда достаточным приближением при описании реальных систем. С математической точки зрения задачи, возникающие при рассмотрении разупорядоченных систем, гораздо сложнее, чем аналогичные задачи, соответствуюшие упорядоченным системам, и характеризуются отсутствием хорошо обоснованных универсальных подходов к их решению. В особенно интересных случаях сильно разупорядоченных систем построение приближенных решений затруднено отсутствием малых параметров. По этим причинам для каждой конкретной задачи, как правило, приходится разрабатывать свой метод решения.

* Всероссийский научный центр "Государственный оптический институт им. С. И. Вавилова", Санкт-Петербург, Россия. E-mail: gkozlov@photonics.phys.spbu.ru 
Задач, решаемых точно, в физике разупорядоченных систем очень мало. Даже предложить более или менее последовательный подход к решению удается лишь для простейших моделей разупорядоченных систем, две из которых и рассматриваются ниже. Это модели одномерного экситона Френкеля при бинарном и равномерном диагональном беспорядках в приближении ближайшего соседа. Рассмотрим цепочку двухуровневых атомов, в которой расщепление $\varepsilon(r)$ уровней атома с номером $r$ может быть $\mathrm{c}$ вероятностью $c$ равно $u$ и с вероятностью $1-c$ равно $-u$ (бинарный беспорядок) или равновероятно распределено в интервале $[-u, u]$ (равномерный беспорядок). Между атомами имеется взаимодействие $\Delta$, способное переносить возбуж дение с одного атома на другой, причем это взаимодействие отлично от нуля только для ближайших соседей. Если число атомов в цепочке равно $N$, то задача сводится к исследованию случайной матрицы гамильтониана $\mathbf{H}$ [1] с элементами

$$
H_{r, r^{\prime}}=\varepsilon(r) \delta_{r, r^{\prime}}+\Delta\left(\delta_{r, r^{\prime}-1}+\delta_{r, r^{\prime}+1}\right), \quad r, r^{\prime}=1,2, \ldots, N
$$

Представляет интерес любая проверенная нетривиальная информация о спектре и собственных векторах этой матрицы. Обычно изучается плотность состояний этой матрицы, в которой диагональные элементы $\varepsilon(r)$ считаются независимыми случайными величинами, имеюшими функцию распределения $P(\varepsilon)$. Для бинарного и равномерного беспорядков эта функция имеет вид соответственно

$$
\begin{aligned}
& P(\varepsilon)=c \delta(\varepsilon-u)+(1-c) \delta(\varepsilon+u), \\
& P(\varepsilon)= \begin{cases}(2 u)^{-1}, & \varepsilon \in[-u, u], \\
0 & \text { в остальных случаях. }\end{cases}
\end{aligned}
$$

Как правило, эту задачу рассматривают в термодинамическом пределе $N \rightarrow \infty$. Заметим, что задача, соответствуюшая бинарному беспорядку, возникает при рассмотрении колебательного спектра изотопически разупорядоченной цепочки, причем существует реальная физическая система [2], [3], описывающаяся этой моделью. Задачи этого типа рассматривались Дайсоном [4], которому удалось получить точное решение для некоторого специального (не бинарного и не равномерного) вида беспорядка. В настояшей работе подход Дайсона применяется к случайным матрицам с бинарным и равномерным беспорядками.

Всюду ниже мы будем исследовать функцию Грина, построенную по гамильтониану (1),

$$
\mathbf{G}=(E-\mathbf{H})^{-1} .
$$

Во всей матрице функции Грина (3) для рассматриваемых задач оказывается особенно важным элемент $G_{N, N}$, который мы будем называть краевой функиией Грина (КФГ). Для расчета плотности состояний необходима объемная функиия Грина - достаточно произвольный элемент $G_{r, r}$ такой, что узел $r$ находится настолько далеко от краев цепочки, что среднее $\left\langle G_{r, r}\right\rangle \equiv\langle g(E)\rangle$ не зависит от номера $r$. КФГ обладает следуюшим 
свойством [1]. Рассмотрим цепочку из $N$ атомов, КФГ которой обозначим $\gamma$ и добавим к ней еше один атом с расщеплением $\varepsilon$. КФГ полученной таким образом цепочки из $N+1$ атомов обозначим $\tilde{\gamma}$. Тогда имеет место следуюшее соотношение:

$$
\tilde{\gamma}=\frac{1}{E-\varepsilon-\Delta^{2} \gamma} .
$$

Если известна функция распределения $\rho_{N}(\gamma)$ для цепочки из $N$ атомов, то функция распределения $\rho_{N+1}(\tilde{\gamma})$ для цепочки из $N+1$ атомов может быть получена при помоши (4). При $N \rightarrow \infty$ эти функции должны совпадать, т.е. $\rho_{N}(\gamma)=\rho_{N+1}(\gamma) \equiv \rho(\gamma)$, что дает следуюшее уравнение [1] для предельной функции $\rho(\gamma)$ :

$$
\rho(\gamma) \gamma^{2}=\int d \gamma^{\prime} P\left(E-\Delta^{2} \gamma^{\prime}-\frac{1}{\gamma}\right) \rho\left(\gamma^{\prime}\right) .
$$

Решение этого уравнения в ряде случаев может быть сушественно нерегулярной функцией, на что было указано в работе [5]. Ниже мы увидим, что для случая бинарного беспорядка это действительно так, в отличие от случая равномерного беспорядка, когда решение (5) представляет собой достаточно "хорошую" функцию. Исследование КФГ представляет интерес, главным образом, потому, что, зная функцию распределения КФГ $\rho(\gamma)$, можно вычислить усредненную объемную функцию Грина по любой из следуюших формул [1]:

$$
\begin{aligned}
\langle g(E)\rangle & =\int \frac{d x d y d \varepsilon \rho(x) \rho(y) P(\varepsilon)}{E-\varepsilon-\Delta^{2}(x+y)}, \\
\langle g(E)\rangle & =\frac{d}{d E} \int d \gamma \rho(\gamma) P(\varepsilon) \ln \left(E-\varepsilon-\gamma \Delta^{2}\right) .
\end{aligned}
$$

Для случая бинарного (2а) или равномерного (2б) беспорядков уравнение (5) имеет соответственно вид

$$
\begin{gathered}
\rho(\gamma) \gamma^{2} \Delta^{2}=c \rho\left(\frac{E-u-\gamma^{-1}}{\Delta^{2}}\right)+(1-c) \rho\left(\frac{E+u-\gamma^{-1}}{\Delta^{2}}\right) \\
2 u \gamma^{2} \rho(\gamma)=\int_{l(\gamma)}^{h(\gamma)} \rho(x) d x, \quad h(\gamma) \equiv \frac{E+u-\gamma^{-1}}{\Delta^{2}}, \quad l(\gamma)=\frac{E-u-\gamma^{-1}}{\Delta^{2}} .
\end{gathered}
$$

Основной целью настоящей работы является исследование этих уравнений. Ниже перечислены основные результаты.

В случае бинарного беспорядка:

1. Показано, что функция $\rho(\gamma)$ неаналитична по $\gamma$ и при $E>E_{\mathrm{c}}(u)$ (формула для вычисления $E_{\mathrm{c}}(u)$ приведена) обрашается в нуль в области числовой оси, представляющей собой совокупность бесконечного числа запрещенных отрезков, разделенных интерва-

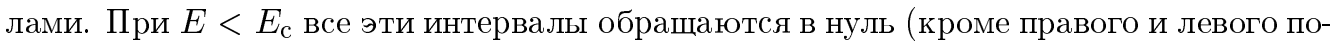
лубесконечных интервалов) и $\rho(\gamma)$ становится отличной от нуля всюду внутри одного отрезка (указанного явно). 


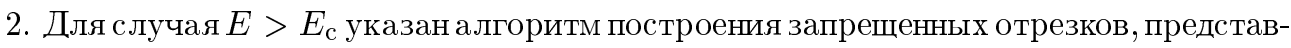
ляющий собой последовательность этапов. На первом этапе определяется область $R_{1}$, в которой $\rho(\gamma)$ заведомо равна нулю. На втором этапе в дополнительной к $R_{1}$ области указывается область $R_{2}$, где $\rho(\gamma)$ также обрашается в нуль. На третьем этапе в области, дополнительной к $R_{1} \cup R_{2}$, определяется область, где $\rho(\gamma) \equiv 0$ и т.д.

3. Каждый этап характеризуется появлением некоторого числа неперекрываюшихся областей, в которых $\rho(\gamma)$ не всюду равна нулю. На каждом этапе определяются гранишы этих областей, а также интегралы от функции $\rho(\gamma)$ по каждой из них. Таким образом, после каждого этапа имеется возможность приближенного расчета средних с функцией $\rho(\gamma)$ путем замены ее в указанных областях на постоянную. От этапа к этапу точность вычисления средних возрастает.

4. Предлагаемый алгоритм построения $\rho(\gamma)$ позволяет вычислять средние с возмож-

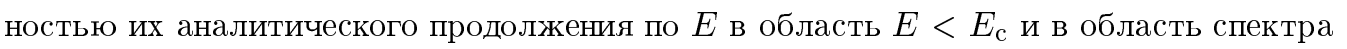
гамильтониана (1). Вычислены $\operatorname{Im}\langle\gamma(E)\rangle$ (плотность возбуждений на краю цепочки) и $\operatorname{Im}\langle g(E)\rangle$ (плотность состояний). Результаты согласуются с результатами, полученными с помощью компьютерной диагонализации.

В случае равномерного беспорядка:

1. Показано, что на некотором (указанном явно) отрезке $F$ функция $\rho(\gamma)$ находится точно и определяется формулой $\rho(\gamma)=1 /\left(2 u \gamma^{2}\right)$.

2. Указаны отображения $\widehat{O}_{ \pm}$, которыми отрезок $F$ переводится в два других отрезка $F_{ \pm}^{\prime}$, на которых $\rho(\gamma)$ определяется с точностью до одной константы. Каждый отрезок $F_{ \pm}^{\prime}$ отображениями $\widehat{O}_{ \pm}$переводится в два следуюших, на каждом из которых $\rho(\gamma)$ вычисляется с точностью до двух констант и т.д.

3. Предложен метод построения $\rho(\gamma)$ во всей области определения, который хорошо согласуется с компютерными расчетами $\rho(\gamma)$. В рамках предложенного метода для усредненной КФГ получено простое замкнутое трансцендентное уравнение, а для границ спектра гамильтониана (1) - аналитическая формула. Вычисленные усредненная КФГ и границы спектра хорошо согласуются с результатами, полученными компютерной диагонализацией и усреднением.

\section{2. БИНАРНЫЙ БЕСПОРЯДОК. ПОСТРОЕНИЕ ФУНКЦИИ РАСПРЕДЕЛЕНИЯ КФГ}

КФГ представляет собой случайную величину, значения которой можно получать следующим образом. Возьмем произвольную цепочку, КФГ которой равна $\gamma_{0}$, и будем добавлять к ней атомы со случайным расшеплением $\varepsilon$. При этом изменение КФГ можно представить как последовательность отображений (4),

$$
\gamma_{n+1}=\frac{1}{E-\varepsilon-\Delta^{2} \gamma_{n}}
$$

со случайным параметром $\varepsilon$. При бесконечном повторении этого отображения величины $\gamma_{n}$ распределяются в соответствии с искомой функцией распределения $\rho(\gamma)$. Если 
бы параметр $\varepsilon$ не флуктуировал, а был бы равен $u$ (или $-u$ ), то отображение (8) быстро сошлось бы к величинам $\gamma_{-}$(или $\left.\gamma_{+}\right)$, определяемым уравнением

$$
\gamma_{ \pm}=\frac{1}{E \pm u-\Delta^{2} \gamma_{ \pm}}
$$

решение которого задается формулой

$$
\gamma_{ \pm}=\frac{E \pm u-\sqrt{(E \pm u)^{2}-4 \Delta^{2}}}{2 \Delta^{2}}
$$

Нетрудно показать (графически, как это обычно делается при изучении аттракторов отображений типа (8)), что если при некотором $n$ величина $\gamma_{n}$ оказалась заключенной между $\gamma_{-}$и $\gamma_{+}$, то $\gamma_{n+1}$ оказывается также заключенной в этом интервале, если $-u<\varepsilon<u$. Так как $\gamma_{n}$ рано или поздно всегда попадет в интервал $\left[\gamma_{+}, \gamma_{-}\right]$, то и все следующие $\gamma_{m}, m>n$, также попадут в этот интервал. Отсюда можно сделать вывод, что функция $\rho(\gamma)$ не равна нулю только в этом интервале, т.е.

$$
\rho(\gamma) \neq 0 \text { при } \gamma \in\left[\gamma_{+}, \gamma_{-}\right]
$$

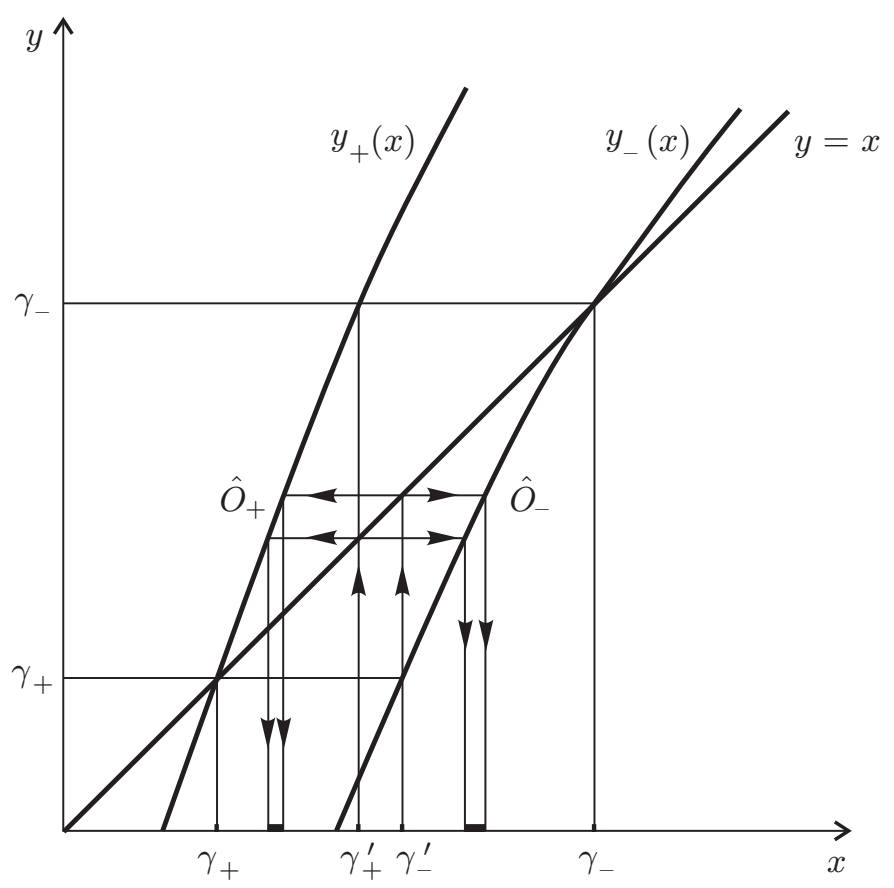

Рис. 1 
Все сказанное выше в равной степени справедливо для бинарного и равномерного беспорядков. Специфика бинарного беспорядка заключается в том, что внутри интервала $\left[\gamma_{+}, \gamma_{-}\right]$можно указать область, где $\rho(\gamma) \equiv 0$. На рис. 1 на плоскости $x y$ приведены графики зависимостей

$$
y_{ \pm}(x)=\frac{E \pm u-x^{-1}}{\Delta^{2}}
$$

представляюшие собой аргументы функций $\rho$ в правой части (7a), а также прямая $y=x$. Определим $\gamma_{ \pm}^{\prime}$ из уравнений

$$
y_{ \pm}\left(\gamma_{ \pm}^{\prime}\right)=\gamma_{\mp}
$$

и построим соответствуюшие точки так, как это показано на рис. 1. Видно, что если $\gamma \in$ $\left[\gamma_{+}^{\prime}, \gamma_{-}^{\prime}\right]$, то $y_{-}(\gamma)<\gamma_{+}$и $y_{+}(\gamma)>\gamma_{-}$, т.е. аргументы функций $\rho$ в правой части $(7 \mathrm{a})$ не принадлежат отрезку $\left[\gamma_{+}, \gamma_{-}\right]$, правая часть (7а) обрашается в нуль и, следовательно,

$$
\rho(\gamma) \equiv 0 \text { при } \gamma \in\left[\gamma_{+}^{\prime}, \gamma_{-}^{\prime}\right]
$$

При этом $\rho(\gamma)$, во всяком случае, не всюду равна нулю при $\gamma \in\left[\gamma_{ \pm}^{\prime}, \gamma_{ \pm}\right]$.

Проинтегрируем теперь уравнение $(7 \mathrm{a})$, например, по промежутку $\left[\gamma_{+}, \gamma_{+}^{\prime}\right]$ :

$$
\int_{\gamma_{+}}^{\gamma_{+}^{\prime}} \rho(x) d x=\frac{1-c}{\Delta^{2}} \int_{\gamma_{+}}^{\gamma_{+}^{\prime}} \rho\left(\frac{E+u-x^{-1}}{\Delta^{2}}\right) \frac{d x}{x^{2}}=(1-c) \int_{\gamma_{+}}^{\gamma_{-}} \rho(x) d x=1-c .
$$

Здесь мы воспользовались тем, что аргумент $y_{-}(\gamma)$ первой функции в правой части (7a) не принадлежит $\left[\gamma_{+}, \gamma_{-}\right]$при $\gamma \in\left[\gamma_{+}, \gamma_{+}^{\prime}\right]$ и, следовательно, интеграл от первой функции равен нулю. Аналогично получается

$$
\int_{\gamma_{-}^{\prime}}^{\gamma_{-}} \rho(x) d x=c
$$

Таким образом, на этом этапе мы можем искомую функцию распределения $\rho(\gamma)$ приближенно представить в виде

$$
\rho(\gamma) \approx \begin{cases}c\left(\gamma_{-}-\gamma_{-}^{\prime}\right)^{-1}, & \gamma \in\left[\gamma_{-}, \gamma_{-}^{\prime}\right] \\ (1-c)\left(\gamma_{+}^{\prime}-\gamma_{+}\right)^{-1}, & \gamma \in\left[\gamma_{+}, \gamma_{+}^{\prime}\right] \\ 0 & \text { в остальных случаях. }\end{cases}
$$

Для дальнейшего удобно ввести понятия двух видов отображений $\widehat{O}_{ \pm}$точек или отрезков. Будем считать, что $x^{\prime}=\widehat{O}_{ \pm} x$, если $y_{ \pm}\left(x^{\prime}\right)=x$. Например, $\widehat{O}_{+} \gamma_{-}=\gamma_{+}^{\prime}$ или $\widehat{O}_{-} \gamma_{+}=\gamma_{-}^{\prime}$ (рис. 1). Аналогично для отрезков. Если отрезок представлять столбцом, верхний и нижний элементы которого суть верхняя и нижняя границы этого отрезка, то

$$
\widehat{O}_{ \pm}\left(\begin{array}{c}
h \\
l
\end{array}\right)=\left(\begin{array}{c}
\widehat{O}_{ \pm} h \\
\widehat{O}_{ \pm} l
\end{array}\right)=\left(\begin{array}{c}
\left(E \pm u-\Delta^{2} h\right)^{-1} \\
\left(E \pm u-\Delta^{2} l\right)^{-1}
\end{array}\right)
$$


Рассуждения, аналогичные приведенным выше для получения условия (12), приводят к следующему утверждению. Если функиия $\rho(\gamma)$, определяемая уравнением (7a), обращается в нуль на некотором отрезке $[a, b]$, то она равна нулю также и на двух отрезках, полученных из $[a, b]$ отображсениями $\widehat{O}_{ \pm}$. Например, равенство нулю $\rho(\gamma)$ на $\left[\gamma_{+}^{\prime}, \gamma_{-}^{\prime}\right]$ приводит к равенству нулю $\rho(\gamma)$ на отрезках, полученных из него отображениями $\widehat{O}_{ \pm}$. На рис. 1 эти отрезки показаны жирными черными линиями, там же стрелками показан графический смысл отображений $\widehat{O}_{ \pm}$. Таким образом, применяя отображения $\widehat{O}_{ \pm} n$ раз или проходя $n$ этапов, из отрезка $\left[\gamma_{+}^{\prime}, \gamma_{-}^{\prime}\right]$ мы получим $2^{n}$ других, где $\rho(\gamma) \equiv 0$. Назовем эти отрезки запрещенными (всего запрешенных отрезков после $n$ этапов будет $\left.2^{n}+1\right)$.

Наряду с запрещенными отрезками, где $\rho(\gamma) \equiv 0$, возникают дополнительные к ним разрешенные отрезки, внутри которых $\rho(\gamma)$ не всюду равна нулю. После $n$ этапов мы получим $2^{n+1}$ разрешенных отрезков (рис. 1 ). Будем характеризовать каждый разрешенный отрезок на $n$-м этапе столбцом, первый и второй элементы которого суть верхняя и нижняя границы разрешенного отрезка, а третий элемент - интеграл от функции $\rho(\gamma)$ по этому разрешенному отрезку.

Пусть пройдено $n$ этапов и имеется $2^{n+1}$ разрешенных отрезков,

$$
\left(\begin{array}{c}
h_{i} \\
l_{i} \\
I_{i}
\end{array}\right), \quad i=1, \ldots, 2^{n+1} .
$$

Тогда нетрудно проверить, что $(n+1)$-й этап заключается в том, что из этой совокупности разрешенных отрезков получается новая совокупность, состояшая из следующих двух классов. Первый класс получается применением отображения $\widehat{O}_{+}$к двум верхним компонентам столбцов совокупности (17) и умножением на $1-c$ третьего компонента, второй класс - применением отображения $\widehat{O}_{-}$к двум верхним компонентам столбцов совокупности (17) и умножением на $c$ третьего компонента, т.е.

$$
\begin{aligned}
& \left(\begin{array}{c}
\tilde{h}_{i} \\
\tilde{l}_{i} \\
\tilde{I}_{i}
\end{array}\right)=\left(\begin{array}{c}
\widehat{O}_{+} h_{i} \\
\widehat{O}_{+} l_{i} \\
(1-c) I_{i}
\end{array}\right)=\left(\begin{array}{c}
\left(E+u-\Delta^{2} h_{i}\right)^{-1} \\
\left(E+u-\Delta^{2} l_{i}\right)^{-1} \\
(1-c) I_{i}
\end{array}\right), \quad i=1, \ldots, 2^{n+1}, \\
& \left(\begin{array}{c}
\tilde{h}_{i+2^{n+1}} \\
\tilde{l}_{i+2^{n+1}} \\
\tilde{I}_{i+2^{n+1}}
\end{array}\right)=\left(\begin{array}{c}
\widehat{O}_{-} h_{i} \\
\widehat{O}_{-} l_{i} \\
c I_{i}
\end{array}\right)=\left(\begin{array}{c}
\left(E-u-\Delta^{2} h_{i}\right)^{-1} \\
\left(E-u-\Delta^{2} l_{i}\right)^{-1} \\
c I_{i}
\end{array}\right), \quad i=1, \ldots, 2^{n+1} .
\end{aligned}
$$

Проверка выполняется по аналогии с формулами (13), (14). Аналогично формуле (15) после $n$ этапов функцию $\rho(\gamma)$ можно приближенно представить в виде

$$
\rho(\gamma)=I_{i}\left(h_{i}-l_{i}\right)^{-1} \text { при } \gamma \in\left[l_{i}, h_{i}\right] .
$$

Алгоритм (18), (19) в сочетании с начальными условиями

$$
\left(\begin{array}{c}
h_{1} \\
l_{1} \\
I_{1}
\end{array}\right)=\left(\begin{array}{c}
\gamma_{+}^{\prime} \\
\gamma_{+} \\
1-c
\end{array}\right), \quad\left(\begin{array}{c}
h_{2} \\
l_{2} \\
I_{2}
\end{array}\right)=\left(\begin{array}{c}
\gamma_{-} \\
\gamma_{-}^{\prime} \\
c
\end{array}\right)
$$




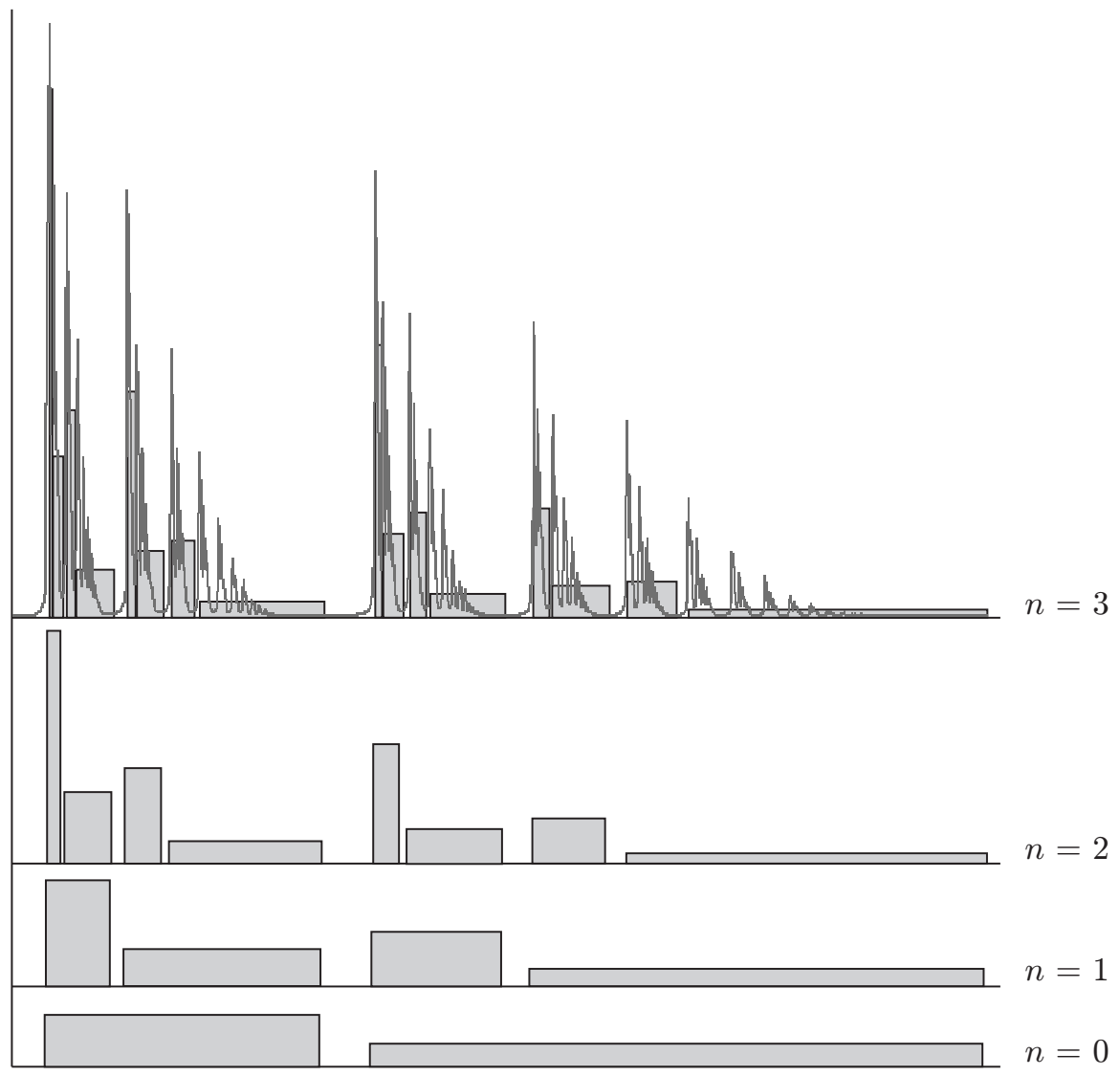

Рис. 2

позволяет строить функцию распределения $\rho(\gamma)$ с произвольной точностью. Пример такого построения при $c=0.5, \Delta=2, u=0.6, E=2 \Delta+u$ показан на рис. 2 (для $n=$ $0,1,2,3)$, где также показана функция $\rho(\gamma)$, полученная компьютерным моделированием (для $n=3$ ).

Описанная выше процедура последовательного дробления разрешенных отрезков напоминает процедуру построения фрактала. Функцию $\rho(\gamma)$ можно рассматривать как “аттрактор" случайного отображения (8). На рис. 3 показана структура этого "аттрактора", полученная следуюшим образом. K “затравочному" значению $\gamma_{0}=0.5\left(\gamma_{+}\left(E_{0}\right)+\right.$ $\left.\gamma_{-}\left(E_{0}\right)\right) \quad\left(E_{0} \equiv 2 \Delta+u\right) 50000$ раз применялось отображение (8), причем параметр $\varepsilon$ генерировался случайным образом в соответствии с распределением (2a) (при $c=0.7$, $\Delta=2, u=0.6$ (рис. 3а) или $u=0.2$ (рис. 3б)), а энергия $E$ равномерно изменялась в диапазоне $E_{0}<E<1.1 E_{0}$. Полученные 50000 точек $\left(\gamma_{n}, E\right)$ строились на плоскости $(\gamma, E)$. Плотность точек по вертикали при заданном $E$ определяется функцией $\rho(\gamma)$. Также на рис. 3 показаны граничные линии первых восьми разрешенных отрезков, построенных при помоши алгоритма (18). Из рисунка видно, что при достаточно малом 


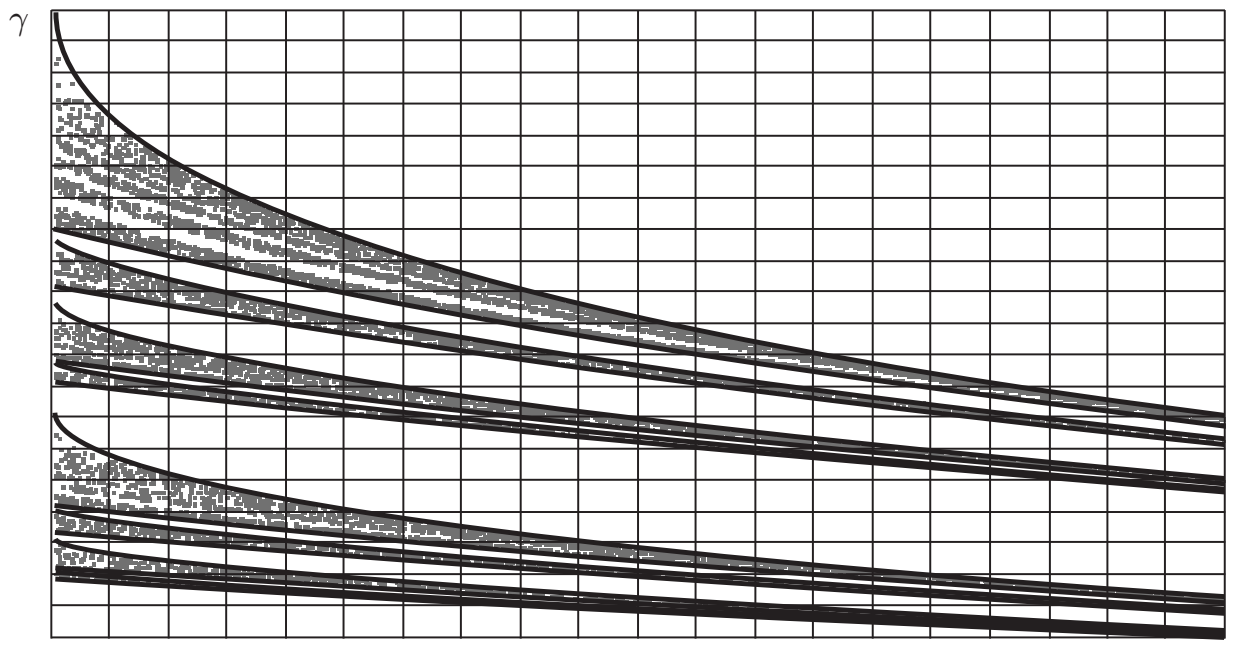

$E_{0}$

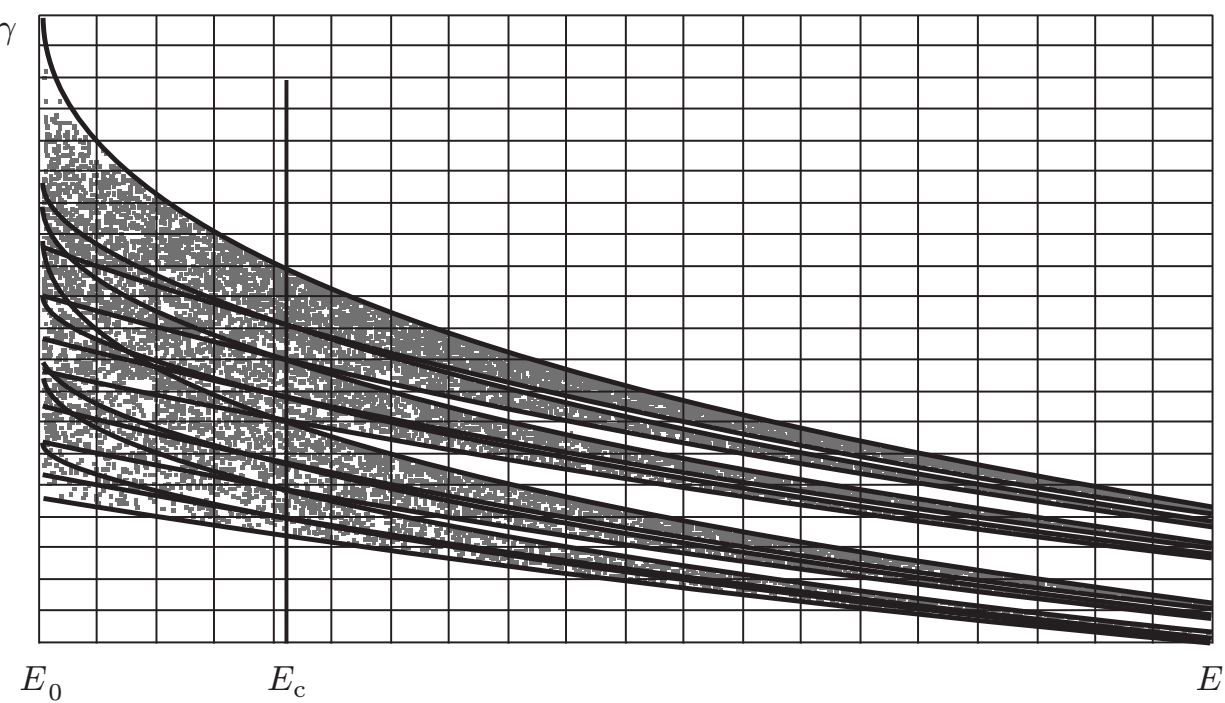

б

Рис. 3

беспорядке $(u=0.2)$, сушествует такое значение энергии $E_{\mathrm{c}}$, при котором запрешенные отрезки исчезают. Значение $E_{\mathrm{c}}$ определяется из уравнения $\gamma_{+}^{\prime}\left(E_{\mathrm{c}}\right)=\gamma_{-}^{\prime}\left(E_{\mathrm{c}}\right)$ или

$$
\sqrt{\left(E_{\mathrm{c}}+u\right)^{2}-4 \Delta^{2}}-\sqrt{\left(E_{\mathrm{c}}-u\right)^{2}-4 \Delta^{2}}=6 u,
$$

которое представляет собой условие исчезновения первого запрещенного отрезка (12). Это влечет за собой исчезновение остальных запрешенных отрезков, которые получа-

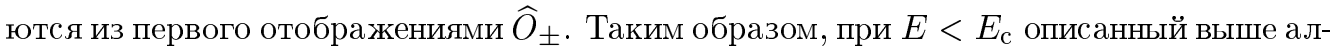


горитм построения $\rho(\gamma)$ нуждается в модификации. Это, однако, совершенно не важно для вычисления усредненных КФГ и объемной функции Грина (6а), которые достаточ-

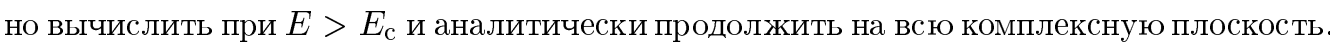
Нетрудно показать, что при $u>\Delta / 4$ величина $E_{\mathrm{c}}$ становится меньше, чем $2 \Delta+u$, т.е. $E_{\mathrm{c}}$ "погружается" в спектр гамильтониана. По-видимому, это "погружение" не связано ни с каким критическим поведением рассматриваемой разупорядоченной системы, однако вопрос этот пока остается открытым. В следуюшем разделе будут описаны вычисления средних КФГ и объемной функции Грина.

\section{3. БИНАРНЫЙ БЕСПОРЯДОК. УСРЕДНЕННЫЕ КФГ И ОБЪЕМНАЯ ФУНКЦИЯ ГРИНА}

Если выполнено $n$ этапов описанного в предыдущем разделе алгоритма построения функции распределения $\rho(\gamma)$, то для усредненной КФГ можно написать следующее приближенное выражение:

$$
\langle\gamma(E)\rangle=\int_{\gamma_{+}}^{\gamma_{-}} \rho(\gamma) \gamma d \gamma \approx \frac{1}{2} \sum_{i=1}^{M} I_{i}\left(h_{i}(E)+l_{i}(E)\right), \quad M \equiv 2^{n+1}
$$

Функции $h_{i}(E)$ и $l_{i}(E)$ аналитичны по $E$, так как они получаются применением аналитических по $E$ отображений $\widehat{O}_{ \pm}(E)$ к аналитическим по $E$ функциям $\gamma_{ \pm}(E)$. Поэтому описанный выше алгоритм допускает аналитическое продолжение по $E$ и с его помощью усредненная КФГ может быть вычислена при любом $Е$.

Несколько более громоздкое, но аналогичное вычисление объемной функции Грина по формуле (6a) приводит к следуюшей приближенной формуле:

$$
\langle g(E)\rangle \approx \sum_{i, j=1}^{M} \frac{I_{i} I_{j}}{\left(h_{i}-l_{i}\right)\left(h_{j}-l_{j}\right)}\left(c \Phi_{i j}(E-u)+(1-c) \Phi_{i j}(E+u)\right),
$$

где

$$
\begin{aligned}
\Phi_{i j}(z) \equiv & \frac{1}{\Delta^{4}}\left\{\left[z-\Delta^{2}\left(l_{i}+l_{j}\right)\right] \ln \left[z-\Delta^{2}\left(l_{i}+l_{j}\right)\right]+\right. \\
& +\left[z-\Delta^{2}\left(h_{i}+h_{j}\right)\right] \ln \left[z-\Delta^{2}\left(h_{i}+h_{j}\right)\right]- \\
& -\left[z-\Delta^{2}\left(h_{i}+l_{j}\right)\right] \ln \left[z-\Delta^{2}\left(h_{i}+l_{j}\right)\right]- \\
& \left.-\left[z-\Delta^{2}\left(l_{i}+h_{j}\right)\right] \ln \left[z-\Delta^{2}\left(l_{i}+h_{j}\right)\right]\right\} .
\end{aligned}
$$

На рис. 4а показаны $\operatorname{Im}\langle g(E)\rangle$ (плотность состояний), полученная компьютерной диагонализацией и усреднением по 10000 реализаций (нижний график), и $\operatorname{Im}\langle g(E)\rangle$, полученная в шесть этапов с помошью описанного выше алгоритма по формуле (21) (верхний график). На рис. 4 приведены $\operatorname{Im}\langle\gamma(E)\rangle$ (плотность возбуждений на конце цепочки), полученная компьютерной диагонализацией и усреднением по 10000 реализаций (нижний график), и $\operatorname{Im}\langle\gamma(E)\rangle$, полученная в десять этапов по формуле (20) (верхний график). 
Здесь и всюду ниже при компьютерной диагонализации использовались случайные матрицы размером $200 \times 200$. Приведенные на рис. 4 а, б зависимости соответствуют случаю сильного беспорядка $(c=0.5, u=1, \Delta=2)$, и функция распределения $\rho(\gamma)$ более или менее однородно заполняет интервал $\left[\gamma_{+}, \gamma_{-}\right]$. Поэтому достаточно небольшого числа этапов, чтобы получить хорошее согласие. Качество зависимостей, полученных по формулам (20), (21), выше, чем полученных путем численной диагонализашии и усреднения, а времени для реализации шести этапов требуется гораздо меньше.

$\operatorname{Im}\langle g(E)\rangle, u=1, \Delta=2, c=0.5, n=6,10000$ реализаций

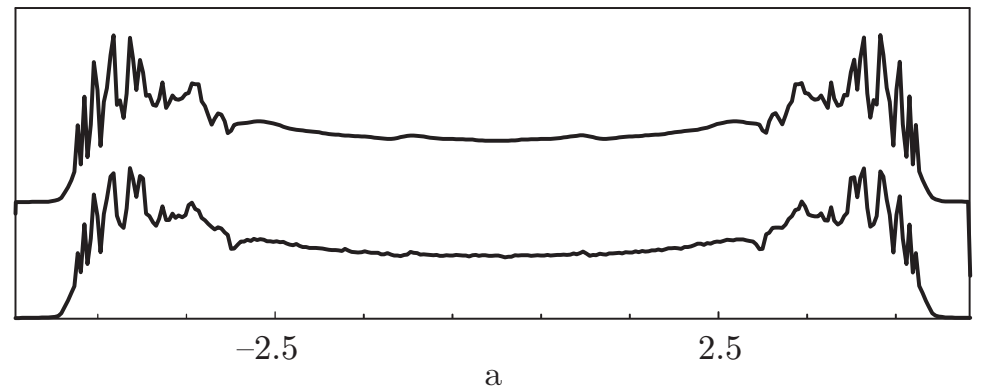

$\operatorname{Im}\langle\gamma(E)\rangle, u=1, \Delta=2, c=0.5, n=10,10000$ реализаций

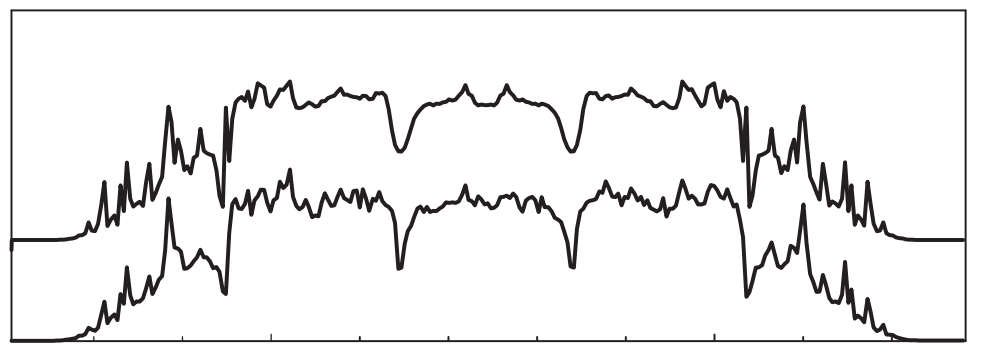

б

$\operatorname{Im}\langle g(E)\rangle, u=1, \Delta=2, c=0.9, n=6,3000$ реализаций

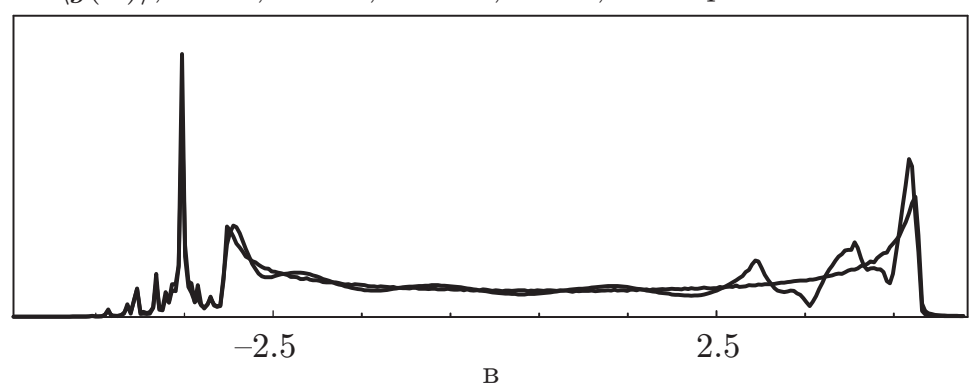

Рис. 4

Согласие несколько ухудшается при переходе к более упорядоченной системе $c=0.9$, $u=1, \Delta=2$, (см. рис. 4в, где изображена плотность состояний $\operatorname{Im}\langle g(E)\rangle$ ). В этом 
случае разрешенные отрезки остаются теми же, что и в предыдущем случае, и требуется большее количество этапов, чтобы сместить максимум $\rho(\gamma)$ к $\gamma_{-}$. Поэтому описанный алгоритм особенно эффективен при расчете сильно разупорядоченных цепочек.

\section{4. ФУНКЦИЯ РАСПРЕДЕЛЕНИЯ КФГ ПРИ РАВНОМЕРНОМ БЕСПОРЯДКЕ}

Как отмечалось выше, при равномерном беспорядке функция $\rho(\gamma)$ отлична от нуля только при $\gamma \in\left[\gamma_{+}, \gamma_{-}\right]$(обозначения те же, что и в разделе 2$)$. Если $\gamma$ такова, что верхний предел интегрирования $h(\gamma)$ в соотношении (7б) больше $\gamma_{-}$, а нижний $l(\gamma)$ меньше $\gamma_{+}$(этому соответствует $\gamma \in\left[\gamma_{+}^{\prime}, \gamma_{-}^{\prime}\right]$, см. рис. 1$)$, то интеграл в (7б) обрашается в единищу и, следовательно,

$$
\rho(\gamma)=\frac{1}{2 u \gamma^{2}} \quad \text { при } \quad \gamma \in\left[\gamma_{+}^{\prime}, \gamma_{-}^{\prime}\right]
$$

Теперь допустим, что $\gamma$ такова, что $\gamma_{+}^{\prime}<h(\gamma)<\gamma_{-}^{\prime}$, т.е. верхний предел интегрирования $h(\gamma)$ в $(7 б)$ находится в области, где функция $\rho(\gamma)$ известна точно из (22). При этом $l(\gamma)<\gamma_{+}$. Следовательно, интегрирование в $(7 б)$ от $\gamma_{+}^{\prime}$ до $h(\gamma)$ может быть выполнено в явном виде,

$$
\rho(\gamma)=\frac{1}{2 u \gamma^{2}} \int_{\gamma_{+}}^{\gamma_{+}^{\prime}} \rho(x) d x+\frac{1}{4 u^{2} \gamma^{2} \gamma_{+}^{\prime}}-\left(\frac{\Delta}{2 u}\right)^{2} \frac{1}{(E+u) \gamma^{2}-\gamma}
$$

при $\gamma_{+}^{\prime}<h(\gamma)<\gamma_{-}^{\prime}$. Нетрудно убедиться в том, что в этом случае $\gamma$ попадает в интервал, полученный из $\left[\gamma_{+}^{\prime}, \gamma_{-}^{\prime}\right]$ отображением $\widehat{O}_{+}$. Таким образом, в этом интервале $\rho(\gamma)$ известна с точностью до константы

$$
S_{+} \equiv \int_{\gamma_{+}}^{\gamma_{+}^{\prime}} \rho(x) d x
$$

Аналогично можно показать, что если $\gamma$ такова, что $\gamma_{+}^{\prime}<l(\gamma)<\gamma_{-}^{\prime}$, т.е. нижний предел интегрирования $l(\gamma)$ в $(7 б)$ находится в области, где функция $\rho(\gamma)$ известна точно из $(22)$, TO

$$
\rho(\gamma)=\frac{S_{-}}{2 u \gamma^{2}}-\frac{1}{4 u^{2} \gamma^{2} \gamma_{-}^{\prime}}+\left(\frac{\Delta}{2 u}\right)^{2} \frac{1}{(E-u) \gamma^{2}-\gamma}
$$

при $\gamma_{+}^{\prime}<l(\gamma)<\gamma_{-}^{\prime}$, где константа

$$
S_{-} \equiv \int_{\gamma_{-}^{\prime}}^{\gamma_{-}} \rho(x) d x
$$

При этом $\gamma \in \widehat{O}_{-}\left[\gamma_{+}^{\prime}, \gamma_{-}^{\prime}\right]$.

Условие нормировки функции $\rho(\gamma)$

$$
S_{+}+S_{-}+\int_{\gamma_{+}^{\prime}}^{\gamma_{-}^{\prime}} \frac{d \gamma}{2 u \gamma^{2}}=1
$$


и определения (11) дают следующее соотношение между $S_{-}$и $S_{+}$:

$$
S_{+}+S_{-}=\frac{\Delta^{2}}{2 u}\left(\gamma_{-}-\gamma_{+}\right)
$$

Продолжая аналогичные построения, можно убедиться в том, что функция $\rho(\gamma)$ может быть определена на отрезках, полученных из $\left[\gamma_{+}^{\prime}, \gamma_{-}^{\prime}\right]$ отображениями $\widehat{O}_{ \pm}$с точностью до все возрастающего числа констант.

Проинтегрируем теперь уравнение (7б) от $\gamma_{+}$до $\gamma_{+}^{\prime}$. При этом в правой части получим $S_{+}$. Интегрируя левую часть по частям, нетрудно показать, что $S_{+}$связана с усредненной КФГ следующим соотношением:

$$
\int_{\gamma_{+}}^{\gamma_{-}} x \rho(x) d x=\langle\gamma(E)\rangle=\gamma_{-}-\frac{2 u}{\Delta^{2}} S_{+} .
$$

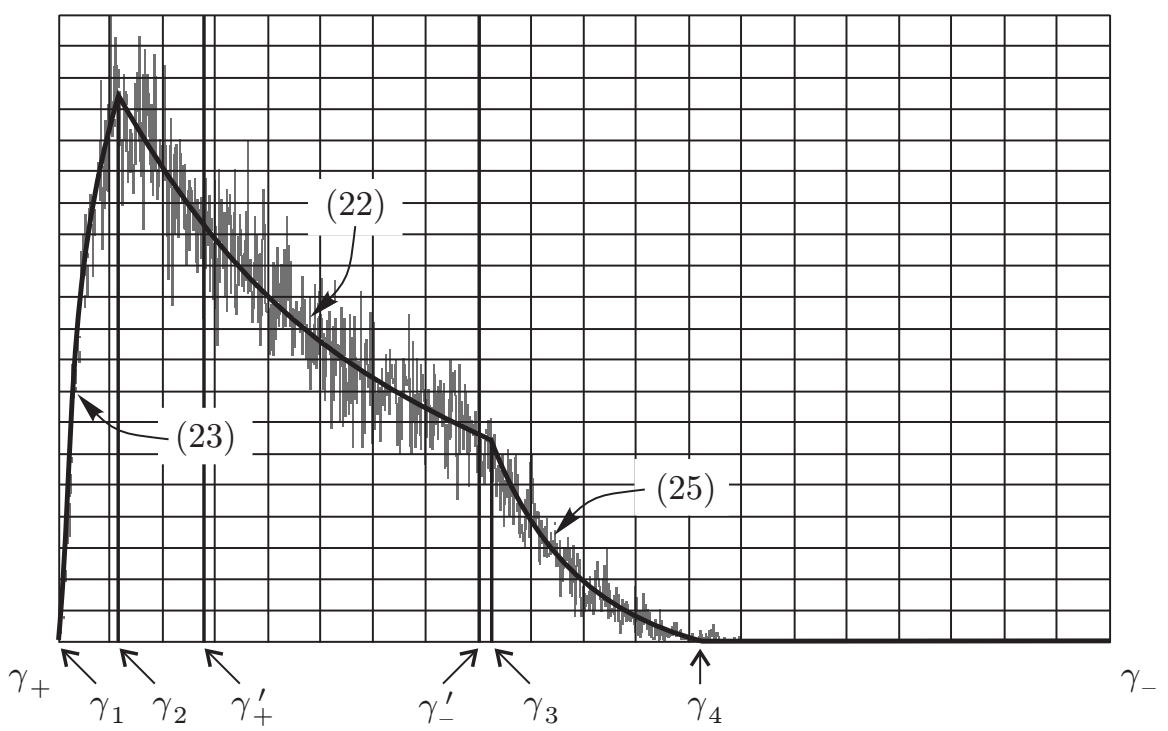

Рис. 5

Ниже мы опишем приближенный метод расчета $S_{+}$. Для этого сделаем следуюшее предварительное замечание. Из уравнения (7б) для равномерного беспорядка следует, что функция $\rho(\gamma)$ непрерывна, а из приведенных выше соображений вытекает, что она аналитична на некоторой совокупности неперекрывающихся отрезков. Таким образом, функция $\rho(\gamma)$ в данном случае не является столь сложной, как в случае бинарного беспорядка, и есть все основания надеяться, что ее можно аппроксимировать несколькими гладкими функциями. В качестве таких функций мы возьмем функции (22), (23), (25). Пусть $\gamma \approx \gamma_{+}$. Из сказанного выше вытекает, что в этом случае $\rho(\gamma)$ естественно аппроксимировать формулой (23), тем более что при некоторых $\gamma$ эта формула является 
точной. При $\gamma \approx 0.5\left(\gamma_{+}+\gamma_{-}\right)$функция $\rho(\gamma)$ определяется формулой $(22)$, а при $\gamma \approx \gamma_{-}$ - формулой (25). Зависимости (22), (23), (25) могут пересекаться с осью $X$ и друг с другом. Обозначим через $\gamma_{1}$ точку пересечения функции (23) с осью $X$, через $\gamma_{2}$ точку пересечения функций (23) и (22), через $\gamma_{3}$ точку пересечения функций $(22)$ и (25) и через $\gamma_{4}$ точку пересечения функции (25) с осью $X$ и построим функцию $\rho(\gamma)$, задавая ее на отдельных отрезках формулами (22), (23), (25),

$$
\rho(\gamma)= \begin{cases}\text { формула }(23), & \gamma \in\left[\gamma_{1}, \gamma_{2}\right], \\ \text { формула }(22), & \gamma \in\left[\gamma_{2}, \gamma_{3}\right], \\ \text { формула }(25), & \gamma \in\left[\gamma_{3}, \gamma_{4}\right], \\ 0 & \text { в остальных случаях. }\end{cases}
$$

Нетрудно определить положение всех точек $\gamma_{i}, i=1, \ldots, 4$, как функций $S_{+}$. После этого соотношение

$$
S_{+}=\int_{\gamma_{1}}^{\gamma_{+}^{\prime}} \rho(x) d x
$$

позволяет получить для $S_{+}$следуюшее трансцедентное уравнение:

$$
\left(\frac{2 u}{\Delta}\right)^{2} S_{+}-\ln \left(1-\frac{2 u \gamma_{+}^{\prime}}{2 u \gamma_{+}^{\prime} S_{+}+1}\right)=2 u \gamma_{-} .
$$

Это уравнение вместе с $(26)$ и (11) позволяет определить $S_{-}$и $S_{+}$и построить $\rho(\gamma)$ в виде (28). Типичный результат приведен на рис. 5 (гладкая кривая), где также показана функция $\rho(\gamma)$, полученная компьютерным моделированием. Параметры $u=1.4$, $\Delta=2, E=2 \Delta+u$. Пользуясь формулой (27), нетрудно получить уравнение, неявно определяющее $\mathrm{K} \Phi \Gamma$ :

$$
\langle\gamma\rangle=\frac{1}{2 u} \ln \frac{E+u-\Delta^{2}\langle\gamma\rangle}{E-u-\Delta^{2}\langle\gamma\rangle} .
$$

Это уравнение дает точные ответы в предельных случаях $u=0$ и $\Delta=0$ и хорошо описывает компьютерные результаты при произвольных $u$ и $\Delta$. На рис. 6 приведен график функции $\operatorname{Im}\langle\gamma(E)\rangle$, полученньй с помошью формулы $(29)$ для $u=\Delta=2$ (гладкая кривая), и график этой же функции, полученный для 5000 реализаций усреднения с помошью компьютерной диагонализации. Уравнение (29) также позволяет в явном виде вычислить границы спектра гамильтониана (1) при равномерном беспорядке (они, очевидно, симметричны). Граничной энергии соответствует исчезновение вешественного решения уравнения (29) - в области спектра КФГ имеет мнимую часть. Несложный графический анализ уравнения (29) показывает, что вешественное решение пропадает, когда его левая и правая части касаются в точке пересечения, т.е.

$$
\begin{gathered}
\frac{d}{d x} \ln \frac{E+u-\Delta^{2} x}{E-u-\Delta^{2} x}=2 u, \\
2 u x=\ln \frac{E+u-\Delta^{2} x}{E-u-\Delta^{2} x} .
\end{gathered}
$$




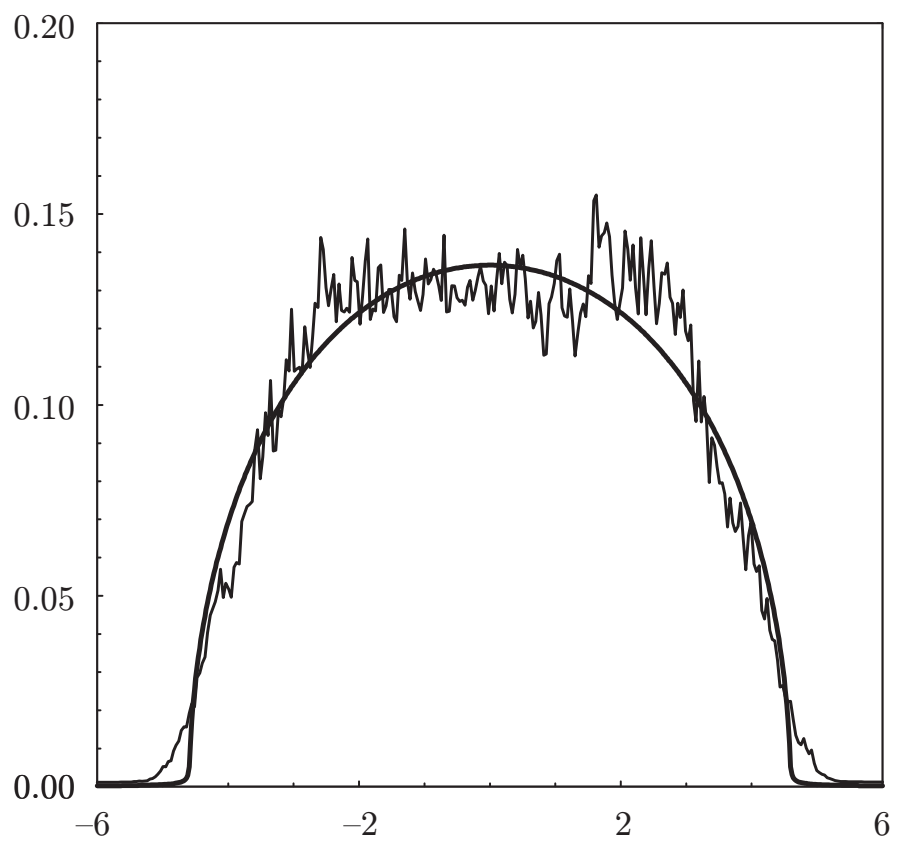

Рис. 6

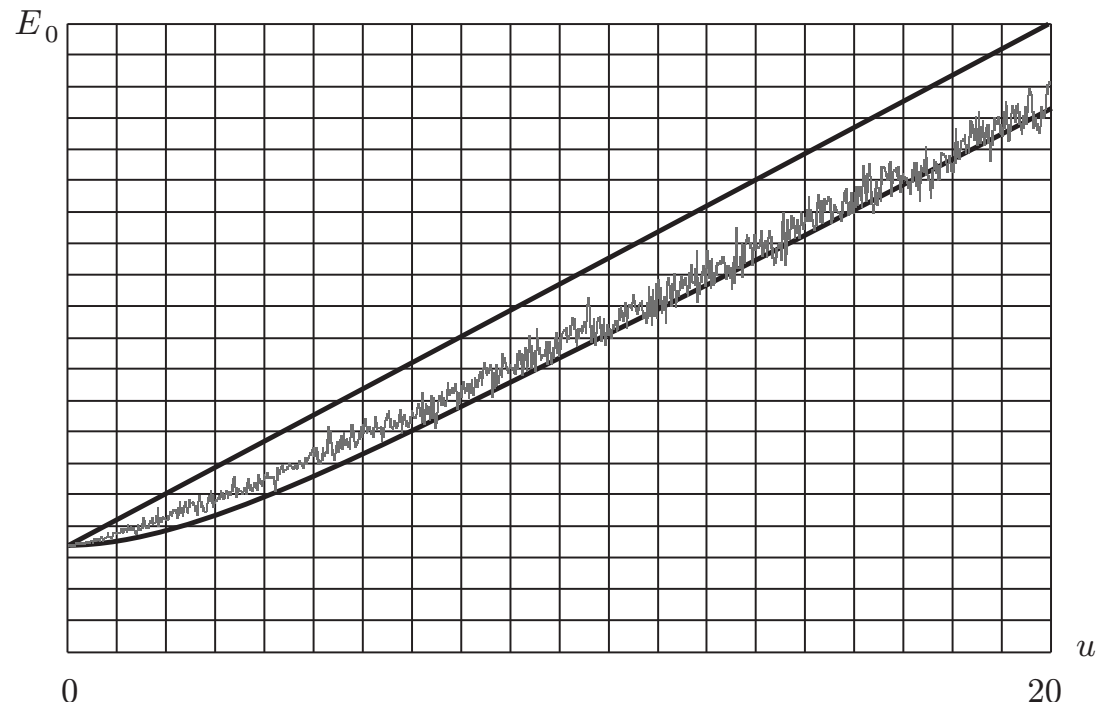

Рис. 7

Из этой системы уравнений определяется граничная энергия для случая равномерного беспорядка

$$
E_{0}=\sqrt{u^{2}+\Delta^{2}}+\frac{\Delta^{2}}{2 u} \ln \frac{\sqrt{u^{2}+\Delta^{2}}+u}{\sqrt{u^{2}+\Delta^{2}}-u} .
$$


На рис. 7 приведено сравнение этой формулы с компютерным расчетом при изменении величины беспорядка $u$ для $\Delta=2$. Гладкая кривая соответствует формуле (31), прямая - формуле (32) (см. ниже), "шумящая кривая" - результат компьютерного моделирования для случая равномерного беспорядка. Видно, что при большой величине беспорядка $u$ ширина спектра не зависит от $\Delta$ - беспорядок "подавляет" межатомное взаимодействие $\Delta$. В этом проявляется отличие равномерно разупорядоченной системы от бинарно разупорядоченной, для которой граница спектра определяется формулой

$$
E_{0}=2 \Delta+u
$$

и включение взаимодействия $\Delta$ вызывает смешение спектра $2 \Delta$ при любом беспорядке.

\section{Список литературы}

[1] И. М. Лифииц, С. А. Гредескул, Л. А. Пастур. Введение в теорию разупорядоченных систем. М.: Наука, 1982.

[2] М. В. Белоусов, Д. Е. Погарев. Письма в ЖЭТФ. 1982. Т. 36. № 5. С. 152.

[3] М. В. Белоусов, Б. Е. Вольф, Е. А. Иванова. Письма в ЖЭТФ. 1983. Т. 38. № 8. С. 376.

[4] F. Dyson. Phys. Rev. 1953. V. 92. P. 1331.

[5] H. Schmidt. Phys. Rev. 1957. V. 105. P. 425.

Поступила в редакцию 21.VII.2003 г., после доработки 16.Х.2003 г. 